\title{
Salienz, Bewertung und Realisierung regionaler Merkmale in Norddeutschland
}

\author{
Yvonne Hettler (Hamburg)
}

\begin{abstract}
While the theoretical concept of perceptual prominence (salience) is subject of various recent studies, there is a lack of empirical evidence concerning the individual prominence of linguistic variables and the perception of these in different regions.

This article presents some results of a study dealing with the perception and production of regional linguistic phenomena in Bremen and Hamburg. A quantitative analysis will be undertaken to investigate which linguistic variables are prominent for speakers and how language attitude and regional localization by non-linguists influence the salience of these phenomena. Also, the correlation between production and perception of linguistic phenomena is discussed by including objective speech data within the context of a qualitative survey. Assuming that the salience of linguistic variables is not fixed, but determined by a variety of factors, it is of interest which different combinations of influencing parameters for perceptual prominence exist.
\end{abstract}

\section{$1 \quad$ Einleitung}

Dass regionale sprachliche Merkmale, die Sprechern stark bewusst sind, nicht unbedingt eine hohe Verbreitung in der Umgangssprache ${ }^{1}$ aufweisen müssen, zeigt sich an den Antworten auf die Frage nach den Eigenschaften des Hamburgischen, die im Rahmen einer Untersuchung zur Hamburger Stadtsprache an die Informanten gestellt wurde: Das am häufigsten genannte Merkmal war, ${ }^{2}$ dass man "über den s-pitzen S-tein s-tolpern" würde (cf. Hettler 2008: 30). Tatsächlich ist das im Anlaut alveolar realisierte /s/ vor Plosiv in Hamburg jedoch kaum noch zu hören (cf. Auer 1998; Lasch 1989) und "zeigt Züge eines Reliktmerkmals, das nur noch von älteren bis alten Sprechern verwendet wird" (Auer 1998: 195). Es scheint aber eines der regionalen Merkmale zu sein, die besonders auffällig sind und derer sich Sprecher und Hörer bewusst sind (cf. zur Bewusstheit ibd.: 181). Dies legt auch eine Untersuchung von Hundt zur Einstellung gegenüber dialektal gefärbter Standardsprache nahe, in der die Audioaufnahme des Hamburgischen nicht als "typisch" Hamburgisch, sondern als "normales Hochdeutsch" charakterisiert wurde, obwohl die Sprachprobe nicht weniger Dialektmerkmale als die anderen Aufnahmen (Bairisch, Pfälzisch, Schwäbisch) enthielt (cf. Hundt 1992: 69). Es scheint, dass die Tonprobe nicht als regional anerkannt wurde, da bestimmte stereotype Erwartungshaltungen bzgl. des Hamburgischen nicht erfüllt wurden, ${ }^{3}$ und zudem gewisse regionale Merkmale nicht wahrgenommen wurden (cf. Hundt 1992: 71). Doch welche vom

\footnotetext{
${ }^{1}$ Unter den Terminus Umgangssprache fallen bei Mihm (2000: 2107): "Sprachverwendungsmuster, die weder der gesprochenen Standardsprache noch den lokalen Basisdialekten zugeordnet werden können, aber Elemente aus beiden Varietätentypen enthalten".

216 von 43 Gewährspersonen.

${ }^{3}$ So kritisierten einige Gewährspersonen an der Aufnahme das Fehlen des alveolaren /s/ (cf. Hundt 1992: 71). Linguistik online 66, 4/14 - http://dx.doi.org/ 10.13092/lo.66.1573
} 
Standard abweichenden Phänomene sind auffällig, also salient, und welche werden nicht bemerkt, sondern als standardsprachlich bewertet?

Während in rezenten Studien das theoretische Konzept "Salienz" des Häufigeren thematisiert wird (cf. besonders Lenz 2010; Purschke 2011; Kiesewalter 2011), ist die empirische Fundierung des Salienzphänomens immer noch ein Desiderat (cf. Elmentaler/Gessinger/Wirrer 2010: 114; Butterworth/Glawe 2011: 373) und es liegen nicht genug "konkrete Untersuchungsergebnisse zur Einzelmerkmalsalienz vor" (Kiesewalter 2011: 336). Insbesondere stellt sich die Frage, ob standarddivergente Phänomene im gesamten Sprachraum ähnlichen Salienzurteilen unterliegen (cf. Schmidt 2005: 301) oder ob bestimmte regionaltypische Varianten für den in der Region ansässigen Sprecher nicht auffällig sind, einem regionsfremden Interaktionspartner aber auffallen würden (cf. Purschke 2011: 73). Die Salienz von Merkmalen müsste somit also auch immer in Bezug auf die Herkunft von Sprecher bzw. Hörer betrachtet werden. Im vorliegenden Beitrag soll ein Projekt zur Wahrnehmung, Bewertung, Verortung und Realisierung regionaler Merkmale in Bremen und Hamburg vorgestellt werden, das weitere Ergebnisse bzgl. der Einzelmerkmalssalienz liefert, die mit den Befunden anderer Studien verglichen werden können. Zudem soll betrachtet werden, wie die Bewertung und regionale Verortung von Varianten Salienz beeinflusst und welchen Zusammenhang es zwischen der Auffälligkeit von Merkmalen und ihrer Realisierung gibt. Allgemein stellt sich die Frage, inwieweit bisherige Annahmen zur Salienz bei der Erklärung der vorliegenden Ergebnisse greifen und ob eine Modifikation des Salienzbegriffs vorgenommen werden muss.

Nach einer kurzen thematischen Einführung zum Thema Salienz und der Erläuterung des Untersuchungsaufbaus sowie zentraler Fragestellungen der Studie werden ausgewählte Ergebnisse der quantitativen Untersuchung thematisiert, wobei der Fokus auf der Wahrnehmung von Phänomenen der phonetisch-phonologischen Ebene liegen wird, jedoch auch einige morpho-syntaktische Merkmale betrachtet werden. ${ }^{4} \mathrm{Im}$ Anschluss an die Darstellung des qualitativen Untersuchungsteils folgen schließlich zwei Beispiele für die individuelle Perzeption und die Produktion regionaler Phänomene.

\section{Salienz}

Im vorliegenden Beitrag wird unter Salienz die "rein (kognitive) Auffälligkeit eines Merkmals" verstanden, die nach Lenz dafür sorgt, "dass ein sprachliches Element aus seinem Kontext hervorgehoben wird und dadurch dem Sprachbewusstsein leichter und schneller zugänglich ist als nicht-saliente Varianten" (Lenz 2010: 94). Bezüglich möglicher Faktoren, die die Auffälligkeit bzw. Unauffälligkeit sprachlicher Merkmale bedingen, gibt es eine Vielzahl an Hypothesen, von denen die für diese Studie am relevantesten kurz vorgestellt werden sollen. ${ }^{5}$

Schirmunski, der die Auffälligkeit von Merkmalen mit seiner Einteilung in primäre und sekundäre Dialektmerkmale, also in stärker und weniger auffallende Abweichungen, in den Fokus nimmt, hält fest, dass auffällige Phänomene sich u. a. durch einen besonders hohen "Grad der artikulatorischen Verschiedenheit" (Schirmunski 1930: 183) auszeichnen. Sie werden zudem am ehesten von nicht heimischen Sprechern angenommen, aber auch am häufigsten (sowohl von heimischen als auch nicht heimischen Sprechern) wieder abgelegt, da sie als Anlass für "Spott" (id. 1928/1929: 168) fungieren können oder zu Verständnisschwierigkeiten führen und somit die überregionale Kommunikation behindern (cf. id. 1930: 172). Die Perzeption des Sprechers bzw. des Hörers ist ein weiteres Kriterium zur Unterteilung in primär

\footnotetext{
${ }^{4}$ Zur Wahrnehmung und Bewertung speziell morpho-syntaktischer Merkmale siehe Hettler (2013).

${ }^{5}$ Für eine ausführlichere Behandlung des Forschungsstandes zur Salienz cf. Lenz 2010; Purschke 2011; Kiesewalter 2011.
} 
und sekundär: Da primäre Merkmale sich durch einen größeren Abstand zur Standardsprache 6 auszeichnen als sekundäre, fallen sie dem Hörer am ehesten auf und sind auch dem Sprecher bewusst. Bei sekundären Merkmalen hingegen ist für den Hörer ein Unterschied zwischen Dialekt und Standardsprache entweder nicht ersichtlich oder er empfindet "das Vorhandensein einer charakteristischen und auffallenden mundartlichen Färbung, kann aber nicht angeben, wodurch sie im einzelnen bedingt ist" (ibd.: 184). Unter dem Einfluss der Schriftsprache werden von den Sprechern zwar die primären Merkmale der gesprochenen Mundart aufgegeben, die sekundären können jedoch aufgrund ihrer perzeptorischen Unauffälligkeit erhalten bleiben (cf. id. 1928/1929: 168). Schirmunski weist somit implizit den sekundären Merkmalen eine "Remanenz"7 in der standardnahen Sprache zu (cf. Lenz 2003: 24).

Schirmunskis Beschreibungskriterien sind eine Mischung aus einerseits objektiven, linguistischen und andererseits eher subjektiven, psycholinguistischen Kriterien (cf. Lenz 2010: 91). ${ }^{8}$ Auch Trudgill (1986) sieht die Gründe für eine erhöhte Auffälligkeit (salience) von Merkmalen einerseits in der strukturellen Beschaffenheit derselben, aber auch in ihrer Bewertung, wenn er schließt, dass Salienz von den Faktoren "stigmatization, linguistic change, phonetic distance and phonological contrast" (ibd.: 37) abhängt. ${ }^{9}$ Mihm (1985) macht die Salienz von Merkmalen hauptsächlich von der Bewertung abhängig, die Varianten im Rahmen der schulischen und außerschulischen Sprachnormvermittlung erhalten (cf. Mihm 1985: 184) und sich durch diese z. B. zu "Fehlerstereotypen" (ibd.: 184) entwickeln. Dies betrifft besonders Merkmale der morpho-syntaktischen Ebene, die in Mihms Untersuchung auffälliger als Standarddivergenzen auf der phonologischen Ebene sind (cf. ibd. 1985: 183-184).

Spiekermann (2008) nimmt an, dass auffällige Merkmale nicht immer stigmatisiert und damit vermieden werden, sondern auch eine positive Bewertung erfahren können, da sie "zum Beispiel [...] als identitätsstiftend empfunden werden" (ibd.: 275).

Bzgl. der Frage, ob Salienz nur von merkmalsintrinsischen Eigenschaften beeinflusst wird und somit eine feststehende Größe ist, ${ }^{10}$ oder auch vom jeweiligen Sprecher, bzw. der Sprechergemeinschaft abhängt, stellt Lenz fest, dass Sprecher saliente Merkmale unterschiedlich wahrnehmen und positioniert den Begriff auf der "subjektiven Ebene" (Lenz 2010: 104).

Auch Purschke (2011) beschäftigt sich mit der subjektiven Relevanz sprachlicher Einheiten, also einer situativen Bedeutung, die der Hörer salienten Merkmalen zugesteht (oder nicht) und von der sein kommunikatives Handeln beeinflusst wird. Diese Faktoren werden unter dem Begriff Pertinenz zusammengefasst (cf. Purschke 2011: 85). ${ }^{11}$ Sprachliche Merkmale können intersubjektiv unterschiedlich bewertet werden und "ohne kommunikative Konsequenzen bleiben" (ibd.: 82) - wie z. B. der Veränderung des Sprachverhaltens bzw. der Wahl bestimmter Varianten (cf. ibd.: 84) - wenn sie "situativ als nicht signifikant und interaktionell als akzeptabel bewertet werden" (ibd.: 85). Auffällige Merkmale werden also nicht automatisch vermieden, selbst wenn sie als normabweichend angesehen werden. Auch die Herkunft des Sprechers bzw. Hörers kann für Purschke Einfluss auf die Auffälligkeit von Merkmalen haben, so dass regionaltypische Varianten Sprechern aus anderen Regionen eher auffallen, als den

\footnotetext{
${ }^{6}$ Bzw. Schriftsprache, da Schirmunski die Auffälligkeit von Merkmalen stets kontrastierend zu dieser bzw. zu anderen Mundarten betrachtet (cf. Schirmunski 1930: 118).

7 Remanente Merkmale sind laut Herrgen/Schmidt "dialektale Merkmale, die Sprecher bei intendierter Standardaussprache trotz hoher metakommunikativer Aufmerksamkeit beibehalten" (Herrgen/Schmidt 1985: 23).

${ }^{8}$ Zur Kritik an Schirmunski, die hier nicht thematisiert werden soll, cf. Trost 1968.

${ }^{9}$ Besonders lexikalische Merkmale sind lt. Trudgill (1986: 25) "highly salient.".

10 Cf. hierzu auch Herrgen/Schmidt (1985: 35), die feststellen, dass die Auffälligkeit von Merkmalen nicht mit der Phonemizität derselben in Zusammenhang stehen muss.

${ }^{11}$ Die Pertinenz bildet neben der Salienz die zweite Kategorie von Hörerurteilen.
} 
einheimischen Sprechern, da diese Merkmale Teil des "individuellen Normhorizonts "Standardsprache" sind" (ibd.: 73). An dieser Stelle ist auch ein möglicher Zusammenhang von Salienz und Gebrauchsfrequenz von Merkmalen miteinzubeziehen, wie er u. a. von Elmentaler/Gessinger/Wirrer (2010) festgestellt wurde. ${ }^{12}$

Insbesondere die Hypothese einer regional unterschiedlichen Auffälligkeit von Varianten ist nur durch den Vergleich verschiedener empirischer Untersuchungen zur Salienz möglich, wobei hierbei die Heterogenität der einzelnen Studien in Bezug auf die verwendete Methode und die Variablenauswahl erschwerend wirkt.

Ergebnisse zur Salienz einzelner Merkmale liegen im deutschsprachigen Raum u. a. von Mihm (1985) zum Rhein-Ruhr-Gebiet, von Kiesewalter (2011) zum Neuhessischen sowie von Lorenz (in diesem Themenheft) für Ostwestfalen vor. Des Weiteren wird für den norddeutschen Raum die Salienz und Gebrauchsfrequenz von regionalen Phänomenen im Rahmen des DFGProjektes "Sprachvariation in Norddeutschland" (SiN) untersucht. ${ }^{13}$ Zum gegenwärtigen Zeitpunkt liegen u. a. Auswertungen einer Vorstudie ${ }^{14}$ zum Projekt (Elmentaler/Gessinger/Wirrer 2010) sowie einer Stichprobenanalyse zu ausgewählten Stimuli aus Ost- und Südwestfalen und Brandenburg (Butterworth/Glawe 2011) vor. ${ }^{15}$ Die Ergebnisse des Projektes sind besonders für die hier vorgestellte Studie relevant, da sich die verwendeten Methoden an die Testbatterie des SiN-Projektes anlehnen. Zudem besteht eine gewisse Schnittmenge in Bezug auf die untersuchten Variablen, die teilweise eine direkte Vergleichbarkeit der Ergebnisse gewährleistet. Im Rahmen der vorliegenden Studie soll zunächst betrachtet werden, inwieweit die Auffälligkeit von Varianten mit einer explizit guten oder schlechten Bewertung sowie der Einordnung in einen bestimmten Sprachraum korreliert ${ }^{16}$ und wie sich Sprecherherkunft und Sprachgebrauch auf die Perzeption von regionalen Merkmalen auswirken.

\section{Untersuchungsaufbau}

Das für diese Studie untersuchte Material entstammt einem Projekt, in dessen Rahmen die Salienz regionaler Merkmale und Parameter, die Salienz bedingen sowie der Zusammenhang zwischen der Auffälligkeit von Varianten und deren Gebrauch untersucht werden. Zu diesem Zweck wird sowohl eine quantitative als auch eine qualitative Analyse durchgeführt.

\subsection{Aufbau und Fragestellungen der quantitativen Untersuchung}

Im Rahmen der quantitativen Untersuchung wurde im Zeitraum von 2010 bis 2012 mit bisher 40 Bremer und 40 Hamburger Sprechern ein Salienztest durchgeführt, dessen Variablen unter 3.3 auszugweise vorgestellt werden. ${ }^{17}$

12 So wurden hochsaliente Merkmale beim Vorlesen von Texten von den untersuchten Gewährspersonen
"tendenziell eher vermieden" (Elmentaler/Gessinger/Wirrer 2010: 120).
13 Siehe zum SiN-Projekt u. a. www.corpora.uni-hamburg.de/sin/index.html.
14 Hierbei handelt es sich um erste Testergebnisse aus Schleswig-Holstein, Brandenburg-Berlin sowie dem
südlichen Niederrhein, wobei auch Daten von Personen ausgewertet wurden, die nicht in das Korpus des SiN-
Projektes eingehen werden (cf. Elmentaler/Gessinger/Wirrer 2010: 116).
15 Eine ausführliche Tabelle zum Forschungsstand zur Salienz findet sich bei Kiesewalter (2011: 365-369).
Hinzuweisen ist noch auf eine frühe Studie von Herrmann-Winter (1977), die implizit die Auffälligkeit von
morpho-syntaktischen Merkmalen im Rahmen einer Studie zum Sprachnormenwandel untersucht, allerdings nicht
in Bezug auf die gesprochene Sprache, sondern auf die Schriftsprache.
16 Es wird hierbei unter Bezugnahme auf u. a. Trudgill und Spiekermann davon ausgegangen, dass sowohl
stigmatisierte als auch prestigehaltige Varianten besonders salient sein können.
17 Bei den Gewährspersonen handelt es sich um 31 Männer und 49 Frauen im Alter von 18-85 Jahren. Es wurde
versucht, bei der Gewährspersonenauswahl darauf zu achten, dass die Sprecher entweder in Bremen bzw.
Hamburg (oder in der direkten Umgebung der Städte) aufgewachsen sind oder seit mindestens 20 Jahren in der
Stadt leben. Im bisherigen Korpus finden sich jedoch vier Gewährspersonen, die weniger als 20 Jahre in Bremen 
Nachdem grundsätzliche biografische Daten der Gewährspersonen (u. a. Alter, Herkunft der Eltern, Schulabschluss, Beruf) sowie die Einschätzung der eigenen Niederdeutsch-Kompetenz erhoben wurden, ${ }^{18}$ folgte der etwa 30-minütige Salienztest. Die Befragten wurden gebeten, anzugeben, was an den gehörten Sätzen für sie kein reines Hochdeutsch (im Sinne, wie es Nachrichtensprecher sprechen sollten) sei - hierbei könne es sich um Merkmale der Aussprache, des Satzaufbaus und um "besondere" Wörter handeln. Um zu verhindern, dass sich die Probanden einem Antwortzwang ausgesetzt fühlten, wurde darauf hingewiesen, dass nicht jeder Satz ein Merkmal enthalten müsse. ${ }^{19}$ Die Sätze wurden nun der Gewährsperson laut vorgespielt. ${ }^{20}$

Bei gehörten Merkmalen wurden Fragen zur regionalen Einordnung, zur Bewertung und zu einer evtl. Korrekturbedürftigkeit der Variante gestellt. ${ }^{21}$ So wurde u. a. versucht, eine mögliche Stigmatisierung bestimmter Phänomene zu eruieren, die auch in Bezug auf höhere bzw. niedrigere Salienz eine Rolle zu spielen scheint (cf. Elmentaler/Gessinger/Wirrer 2010: 112). ${ }^{22}$

Die Ergebnisse der quantitativen Untersuchung ermöglichen es, eine evtl. altersspezifische oder geschlechtsspezifische Wahrnehmung auszumachen, sowie saliente Phänomene zu bestimmen. Des Weiteren können Aufschlüsse über die regionale Zuordnung (seitens der befragten Individuen) bestimmter Merkmale, sowie über die Bewertung salienter Formen gewonnen werden. Die quantitative Untersuchung liefert neben den Perzeptionsdaten vorrangig Einstellungsdaten. Um auch objektive Daten der Sprachproduktion hinzuzuziehen und den Einfluss individueller linguistischer und extralinguistischer Faktoren auf die Perzeption zu eruieren, sollen in der Studie exemplarisch einzelne Gewährspersonen im Rahmen einer qualitativen Untersuchung genauer betrachtet werden.

\subsection{Aufbau und Fragestellungen der qualitativen Untersuchung}

Im Rahmen der qualitativen Untersuchung wurden in Bremen und Hamburg insgesamt 20 weitere Personen befragt, von denen jeweils 5 Personen über 65 sowie 5 Personen unter 35 waren. ${ }^{23}$

Um den Sprachgebrauch der Befragten für eine spätere Variablenanalyse in verschiedenen Formalitätsgraden untersuchen zu können, wurden die Settings Freundesgespräch, Interview sowie Vorlesetext gewählt. ${ }^{24}$ Das Setting Freundesgespräch wurde nicht für jede der 20 Gewährspersonen einzeln erhoben, sondern gruppenweise. Dies bedeutet, dass je 5 befreundete

oder Hamburg gelebt haben, da sie aber zumindest im nord- bzw. niederdeutschen Raum (Emsland, Münster/Westfalen, Ostfriesland und Schleswig-Holstein) aufgewachsen sind, werden sie im Rahmen dieses Beitrages mit einbezogen.

18 Auf die Erhebung von Einstellungsdaten oder auf Fragen zum Hamburgischen und Bremischen wurde hier verzichtet, damit die Gewährspersonen den Salienztest so unvoreingenommen wie möglich ablegen konnten.

19 Tatsächlich wies nur einer der Sätze keine Standarddivergenz auf.

${ }^{20} \mathrm{Zu}$ den Stimuli des Salienztests siehe 3.3.

${ }^{21}$ Die Fragen lauteten: 1. Wo spricht man so?; 2. Wie finden Sie es, wenn jemand so spricht?; 3. Ist das Gehörte für Sie schlechtes oder falsches Deutsch oder würden Sie solche Kategorien hier nicht ansetzen?; 4. Haben Sie den Drang, jemanden zu korrigieren, wenn er so spricht?

22 Eine ausführliche und kritische Methodendiskussion soll in weiterführenden Analysen unter Einbeziehung aller Untersuchungsergebnisse durchgeführt werden. An dieser Stelle sei zunächst vermerkt, dass die Erhebung subjektiver Sprachdaten grundsätzlich einige methodische Probleme mit sich bringt. So erhält man u. a. "Meinungen zu einem Punkt, über den man [der Befragte, Y. H.] zumeist noch gar nicht so genau nachgedacht hat, so dass nicht so ganz klar wird, welche Tatsachen hier besonders fokussiert werden" (cf. Eichinger 2010: 433). Auch besteht die Gefahr, dass Gewährspersonen vom Explorator geäußerte Bezeichnungen einfach übernehmen (cf. Dingeldein 1994: 198). Zu einigen Schwierigkeiten bei der Interpretation von Einstellungsurteilen siehe 4.3.

23 Davon sind 14 männlich und 6 weiblich.

${ }^{24}$ Ein solches Verfahren wurde u. a. im Rahmen der SiN-Erhebungen verwendet (cf. Elmentaler et al. 2006). 
Gewährspersonen bei einem etwa zweistündigen gemeinsamen Treffen ohne die Anwesenheit des Explorators aufgenommen wurden. Einige Zeit nach dem Freundesgespräch wurden Einzelinterviews mit allen Gewährspersonen geführt. Es handelte sich um leitfadengestützte Interviews, die unter anderem Fragen zu Sprachwissen, Sprachbewertung, Ortsloyalität und Normbewusstsein enthielten. Im Anschluss an das Interview wurden die Probanden gebeten, einen Text vorzulesen, um auch die standardnächste, da formellste Sprechlage zu überprüfen (siehe Anhang). Der gewählte Vorlesetext enthält fast alle Salienztest-Stimuli aus dem phonetisch/phonologischen Bereich in, zum Teil, verschiedenen Kontexten. Im Anschluss an den Vorlesetext wurde der Salienztest, der auch schon für die quantitative Untersuchung verwendet wurde, durchgeführt. Auch dieses Gespräch wurde aufgenommen, um die zahlreichen metasprachlichen Kommentare der Gewährspersonen zu sichern.

Die Ergebnisse des Tests sollen zum einen mit den ebenfalls im Interview gewonnenen biografischen Daten korreliert werden, um mögliche Gründe für die individuelle Perzeption zu eruieren und zum anderen sollen die Ergebnisse mit tatsächlich realisierten Formen der Gewährspersonen abgeglichen werden. Zu diesem Zwecke werden Ausschnitte des Freundesgesprächs sowie des Interviews und der gesamte Vorlesetext mithilfe einer variablenbasierten Type-Token-Analyse untersucht. ${ }^{25}$ Dieser liegen die Stimuli des Salienztests zugrunde, welche im Folgenden vorgestellt werden sollen.

\subsection{Ausgewählte Variablen des Salienztests}

Bei den insgesamt 33 Variablen des Salienztests handelt es sich um phonetisch-phonologische, morpho-syntaktische und lexikalische Phänomene der Bremer und Hamburger Stadtsprache. ${ }^{26}$ Die meisten Merkmale sind nicht ausschließlich entweder Hamburg oder Bremen zuzuordnen, sondern betreffen in unterschiedlicher Ausprägung die regional gefärbte Sprache beider Städte oder sind weit verbreitete Merkmale der norddeutschen Umgangssprache, die auch überregional auftreten können. Pro Satz findet sich ein Stimulus, da anzunehmen ist, dass mehrere Stimuli die Aufmerksamkeit des Hörers überfordern würden und bei nur einer Interferenz zudem eine bessere Kontrolle der Merkmale gewährleistet ist (cf. hierzu auch Elmentaler/Gessinger/Wirrer 2010: 115-116). ${ }^{27}$

Auf der phonologischen Ebene wurden neben dem alveolar realisierten /s/ im Anlaut u. a. die Spirantisierung von $/ \mathrm{g} /$ in unterschiedlichen Kontexten, so auch lexemgebunden und in Verbindung mit der Realisierung von vokalisiertem /r/ wie bei der Aussprache von Hamburg als Hambuich geprüft. Des Weiteren werden die Rundung von /i/, der Ausfall einer Affrikatenkomponente, Kurzvokale sowie Lenisierungen untersucht.

Im Bereich der Grammatik wurde u. a. die Pluralendung -en, die Trennung von Pronominaladverbien, die Verwendung der direktiven Präposition nach statt $z u$ sowie der Gebrauch von wie nach Komparativ betrachtet. Im Bereich der Lexik wurde das in Bremen

\footnotetext{
25 Der Type-Token-Analyse werden je 2500 Wörter der Setting Interview und Freundesgespräch sowie der gesamte Vorlesetext (343 Wörter) zugrunde gelegt.

26 Die Auswahl erfolgte auf Basis von u. a. Auer 1998, Martens 1981, Scheel 1963, Stellmacher 1977 und 1981. Das Lexem $u m z u$ wird vor allem in populärwissenschaftlicher Literatur als in Bremen gebräuchlich eingeordnet, cf. hierzu z. B. Kellner 2011.

27 Da zudem anzunehmen ist, dass die Perzeption eines Merkmals auch vom Satzaufbau und der Stellung des Stimulus innerhalb des Satzes abhängt, wurde bei der Konstruktion der Sätze versucht, die Stimuli nicht unbedingt an prominenten Stellen des Satzes wie dem Anfang oder Ende zu platzieren, und die Sprecherin, die die Stimuli eingesprochen hat, wurde angewiesen, möglichst wenig zu betonen. Trotzdem ist der Einfluss des jeweiligen Testsatzes auf die Wahrnehmung eines Merkmals nicht ganz auszuschließen (siehe auch 4.1). Da jedoch einige Stimuli in z. T. anderen Kontexten auch in Salienztests im Rahmen einer Voruntersuchung (cf. Hettler 2008) sowie im Rahmen einer universitären Veranstaltung (Nacht des Wissens 2011, befragt wurden insgesamt 78 Personen, darunter 48 Hamburger) verwendet wurden, ist es in weiterführenden Analysen möglich, ausschnittsweise zu überprüfen, ob die Salienz eines einzelnen Merkmals je nach Testsatz variiert.
} 
gebräuchliche Lexem umzu in einen Satz integriert. Die folgende Tabelle zeigt eine Auswahl von vierzehn untersuchten Variablen mit den jeweiligen Salienztestsätzen. ${ }^{28}$

\begin{tabular}{|c|c|c|}
\hline Ebene & Phänomen & Salienztestsatz \\
\hline \multirow[t]{10}{*}{ Phonologie } & $\begin{array}{l}\text { Spirantisierung } / \mathrm{g} /+ \\
\text { Vokalisierung } / \mathrm{r} / \text { bei -burg }\end{array}$ & $\begin{array}{l}\text { Das Schönste an Hambuich sind die Alster und } \\
\text { das Rathaus. }\end{array}$ \\
\hline & $\begin{array}{l}\text { Spirantisierung } / \mathrm{g} / \mathrm{im} \\
\text { gedeckten Auslaut }\end{array}$ & $\begin{array}{l}\text { Warum hast Du mir nicht gesacht, dass Du } \\
\text { Geld brauchst? }\end{array}$ \\
\hline & $\begin{array}{l}\text { Spirantisierung /g/ im } \\
\text { Auslaut }\end{array}$ & $\begin{array}{l}\text { Können Sie mir den kürzesten Wech zum } \\
\text { Bahnhof beschreiben? }\end{array}$ \\
\hline & Alveolar realisiertes $/ \mathrm{s} /$ & $\begin{array}{l}\text { Genau an diesem Ort haben sie S-törtebeker } \\
\text { hingerichtet. }\end{array}$ \\
\hline & Vokalkürzung /a:/ & Die Kinder lieben es, im Grass zu spielen. \\
\hline & Rundung /i/ & Wir sind jeden Tag schwümmen gegangen. \\
\hline & $\begin{array}{l}\text { Ausfall } \\
\text { Affrikatenkomponente }\end{array}$ & $\begin{array}{l}\text { Auf dem Bauernhof gibt es Hühner, Ferde und } \\
\text { Kühe. }\end{array}$ \\
\hline & Lenisierung $/ \mathrm{t} /$ & $\begin{array}{l}\text { Die Feuerwehr schickte zehn } \\
\text { Reddungsfahrzeuge aus. }\end{array}$ \\
\hline & Lenisierung $/ \mathrm{k} /$ & Ich habe den Wegger auf halb sieben gestellt. \\
\hline & Lenisierung $/ \mathrm{p} /$ & $\begin{array}{l}\text { Wir sollten die Thesen auf eine große Pabbe } \\
\text { schreiben. }\end{array}$ \\
\hline \multirow[t]{3}{*}{$\begin{array}{l}\text { Morpho- } \\
\text { syntax }\end{array}$} & Pluralbildung auf -en & $\begin{array}{l}\text { Ich hätte gerne fünf Scheiben Brot und drei } \\
\text { Stücken Kuchen. }\end{array}$ \\
\hline & $\begin{array}{l}\text { Direktive Präposition nach } \\
\text { statt } z u\end{array}$ & Ich gehe am Samstagabend nach Müllers. \\
\hline & $\begin{array}{l}\text { Trennung } \\
\text { Pronominaladverb }\end{array}$ & $\begin{array}{l}\text { Es gibt jetzt Studiengebühren? Da habe ich } \\
\text { noch gar nichts von gewusst. }\end{array}$ \\
\hline Lexik & $\begin{array}{l}\text { Bremer Ausdruck für } \\
\text { "drum herum, in der } \\
\text { Umgebung" }\end{array}$ & $\begin{array}{l}\text { In Hamburg und } u m z u \text { gibt es viel zu } \\
\text { entdecken. }{ }^{29}\end{array}$ \\
\hline
\end{tabular}

Tabelle 1: Auswahl der untersuchten Variablen und Testsätze.

\section{$4 \quad$ Quantitative Ergebnisse}

Zunächst sollen die Ergebnisse des Salienztests, der im Rahmen der quantitativen Untersuchung mit 80 Gewährspersonen aus Bremen und Hamburg durchgeführt wurde, thematisiert werden.

\footnotetext{
28 Varianten sind die Standardrealisierung (ausgehend vom Aussprache-Duden 2005 und Grammatik-Duden 2009) und die standarddivergente Realisierung. Bei der Phänomenbeschreibung ist anzumerken, dass die Nichtstandardrealisierung angegeben ist.

${ }^{29}$ Für die Bremer Gewährspersonen lautete der Satz abweichend: "In Bremen und umzu gibt es viel zu entdecken."
} 


\subsection{Salienz insgesamt}

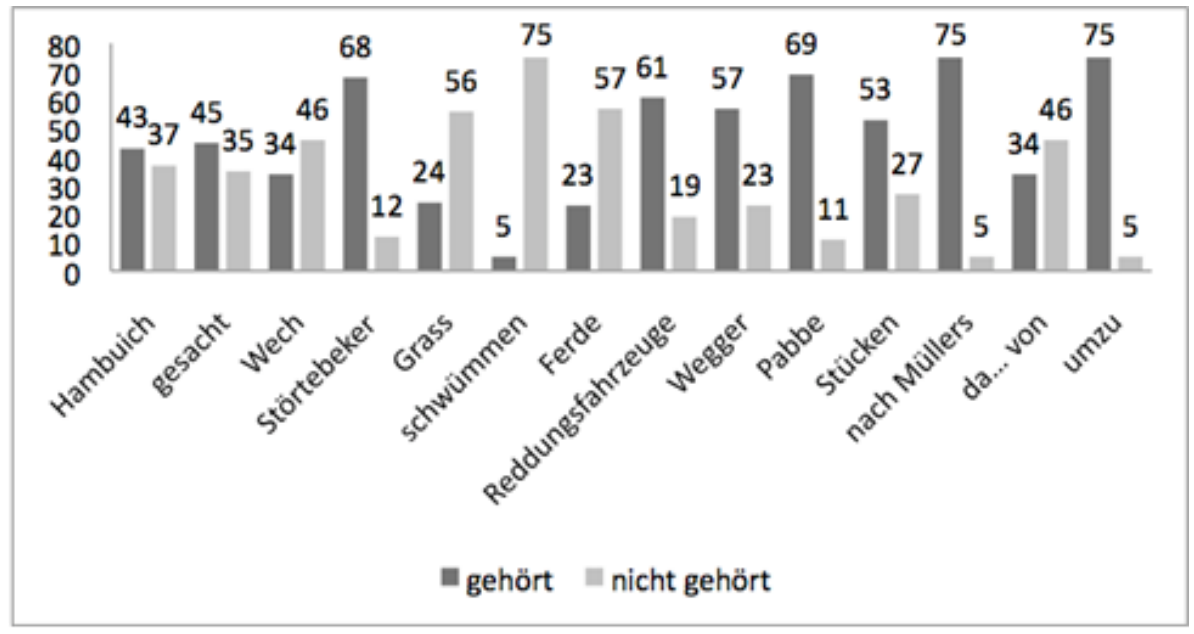

Abbildung 1: Ausgewählte Ergebnisse des Salienztests in absoluten Zahlen (80 GP).

Die Wahrnehmung der Stimuli ist unterschiedlich: Allgemein kann konstatiert werden, dass Merkmale der syntaktischen Ebene (nach Müllers) sowie der lexikalischen Ebene (umzu) insgesamt auffälliger sind als jene der phonologischen Ebene. Dies stützt sowohl Trudgills Annahmen als auch die Ergebnisse von Mihms Studie (cf. Kapitel 2). ${ }^{30}$

Eine Ausnahme stellt die Trennung des Pronominaladverbs davon dar, die nur von 34 der Beteiligten wahrgenommen wurde und somit zu den weniger salienten Merkmalen gehört. ${ }^{31}$ Unter den Merkmalen der phonologischen Ebene ist der Stimulus Pabbe am salientesten und für umgerechnet $86 \%$ der Gewährspersonen auffällig, gefolgt von S-törtebeker mit alveolar realisiertem /s/. Allgemein sind Lenisierungen salienter als Spirantisierungen: Während die Stimuli Wegger und Reddungsfahrzeuge von $71 \%$ bzw. $76 \%$ der Befragten als standarddivergent charakterisiert wurden, fielen nur knapp über der Hälfte der Gewährspersonen die Formen Hambuich und gesacht (54 \% bzw. $56 \%$ ) auf. Die Spirantisierung in Wech wurde sogar nur noch von unter der Hälfte der Befragten (43\%) wahrgenommen. Die unterschiedliche Salienz der drei letztgenannten Varianten spricht dafür, dass die "Salienz einer Variante auch von ihrer Lexemeinbettung und ihrem sprachlichen Kontext abhängt." (Elmentaler/Gessinger/Wirrer 2010: 121).

Kurzvokale, hier in Grass, sowie der Ausfall der Affrikatenkomponente bei Ferde gehören zu den weniger salienten Formen, und werden nur noch von etwa einem Viertel der Gewährspersonen bemerkt. Auch bei Elmentaler/Gessinger/Wirrer gehört der Ausfall der Affrikatenkomponente zu den wenig bis gar nicht salienten Phänomenen - die Abweichung bei Fingsten wurde gar von keiner der 48 Gewährspersonen bemerkt (cf. Elmentaler/Gessinger/Wirrer 2010: 118). ${ }^{32}$ Bei Lorenz (in diesem Themenheft), die im Rahmen ihrer Studie einen Salienztest mit insgesamt 101 Studierenden durchführt, um zu untersuchen, inwieweit sich die Wahrnehmung von einheimischen und zugezogenen Personen in Ostwestfalen unterscheidet, ist die Auffälligkeit des Stimulus Flanze etwas höher als in der

\footnotetext{
${ }^{30}$ Zur Salienzhierarchie aller im Test überprüften Stimuli der morpho-syntaktischen Ebene cf. Hettler (2013: 170).

${ }^{31}$ Dies deckt sich mit den Ergebnissen der Studie von Herrmann-Winter, in welcher der Großteil der Befragten (77,8 \%) die Trennung von davon als standardkonform bewertete (cf. Herrmann-Winter 1977: 243-244).

32 Wie unter 2. vermerkt, handelt es sich hierbei um erste Testergebnisse im Rahmen der Befragungen des Projekts "Sprachvariation in Norddeutschland" (SiN). Ausgewertet wurden die Angaben von 48 Gewährspersonen: 24 Frauen aus Orten in Schleswig-Holstein und dem südlichen Niederrhein sowie 24 Studenten, die im Rahmen eines Seminars in Brandenburg-Berlin befragt wurden. Bei der Auswertung der Daten ging es zunächst u. a. darum, ob unterschiedliche Varianten auch eine unterschiedliche Salienz besitzen und ob diese abhängig von der regionalen Herkunft der Gewährsperson und der Gebrauchshäufigkeit in der Alltagssprache ist (cf. Elmentaler/Gessinger/Wirrer 2010: 117-119).
} 
vorliegenden Studie: Die gemittelte Salienz beträgt bei den untersuchten 32 Zugezogenen aus dem Niederdeutschen Raum 34 \%. ${ }^{33}$

Am wenigsten auffällig ist die Rundung des /i/ in schwümmen, die nur von 5 Personen wahrgenommen wurde.

Die ermittelte Salienzhierarchie der spirantisierten Formen, die in der vorliegenden Studie bzgl. ihrer Auffälligkeit einen mittleren Platz einnehmen, deckt sich nur zum Teil mit den Ergebnissen von Elmentaler/Gessinger/Wirrer (2010), Lorenz (in diesem Themenheft) sowie Mihm (1985), der in seiner Studie zum Prestige und Stigma der Regionalsprache im Ruhrgebiet 100 Gewährspersonen Fragen zur Einstellung gegenüber den Ruhrdeutschen stellt sowie die Auffälligkeit von regionalen Varianten untersucht. ${ }^{34}$ So wurde der Stimulus Kriech von $25 \%$ und der Stimulus gefraacht sogar nur von $13 \%$ der Gewährspersonen als auffällig bezeichnet (cf. Mihm 1985: 183). Bei Elmentaler/Gessinger/Wirrer werden die Formen liecht, gefraacht und Berch dagegen $\mathrm{zu}$ den Varianten mit recht hoher Auffälligkeit gerechnet (cf. Elmentaler/Gessinger/Wirrer 2010: 117-118) ${ }^{35}$ und bei Lorenz beträgt die gemittelte Salienz der Stimuli liecht und Rückwech sogar 89 \% Prozent bei den einheimischen Gewährspersonen aus Ostwestfalen bzw. $97 \%$ bei den beiden Zugezogenengruppen (cf. Lorenz in diesem Themenheft). Die unterschiedlichen Ergebnisse können zum einen durch die Methode bedingt sein, da Mihm, im Gegensatz zu dem in dieser Studie und im SiN-Projekt verwendeten Salienztest, keine isolierten Stimuli verwendet, sondern die Sätze mehrere Abweichungen enthalten (cf. Mihm 1985: 182). Auch bei Lorenz können die Sätze bis zu drei regionale Merkmale aufweisen (cf. Lorenz in diesem Themenheft). Zum anderen ist im Falle von Berch das schon thematisierte lautliche Umfeld einer getesteten Variante mit einzubeziehen. So wäre anzunehmen, dass ein spirantisiertes /g/ im Auslaut nach Liquid auffälliger ist als in anderen Lautkontexten. ${ }^{36}$ Von hoher Relevanz scheint zudem der Einfluss der Herkunft von Gewährspersonen auf die Wahrnehmung regionaler Merkmale zu sein, was sich in den Ergebnissen von Mihm und auch deutlich bei Elmentaler/Gessinger/Wirrer abzeichnet: ${ }^{37}$ Nach Regionen aufgeschlüsselt, wird die Spirantisierung von /g/ nach Langvokal nur von durchschnittlich $56 \%$ der 18 Schleswig-Holsteiner Gewährsfrauen wahrgenommen, während der Stimulus den Befragten in Berlin-Brandenburg sowie am südlichen Niederrhein eher auffiel (cf. Elmentaler/Gessinger/Wirrer 2010: 118). ${ }^{38}$

Im Bereich der Lenisierungen wurde der Stimulus Wegger auch im Rahmen der SiNTestbatterie überprüft. ${ }^{39}$ Auch hier gibt es wieder regionale Unterschiede, die Werte für das lenisierte $/ \mathrm{k} /$ in der vorliegenden Studie decken sich hierbei eher mit den Auffälligkeitswerten aus Brandenburg-Berlin und dem südöstlichen Niederrhein, die bei ca. $50 \%$ liegen, während die Gewährspersonen aus Schleswig-Holstein den Stimulus nur zu 11 \% als standarddivergent

\footnotetext{
$33 \mathrm{Zu}$ bemerken ist, dass dieses Phänomen für die Befragten aus Ostwestfalen sowie aus den mittel- und oberdeutschen Regionen mit einem Salienzmittelwert von 43 \% bzw. 53 \% deutlich auffälliger ist (cf. Lorenz in diesem Themenheft).

34 Die Gewährspersonen stammen zur Hälfte aus Mühlheim und zur Hälfte aus Duisburg und wurden nach Alter, Geschlecht, Schulabschluss und Berufsstatus gestaffelt ausgewählt (cf. Mihm 1985: 165-166).

35 Die Werte liegen zwischen $50 \%$ und $100 \%$.

36 Cf. hierzu auch bei Elmentaler/Gessinger/Wirrer (2010: 122) die Ergebnisse für die Schleswig-Holsteiner Gewährspersonen.

37 In Bezug auf die g-Spirantisierung jedoch nicht bei Lorenz, da die Variante für alle Gewährspersonen hoch auffällig war. Hier wäre zu überlegen inwieweit neben der verwendeten Salienztestmethode auch die Auswahl der Gewährspersonen die Ergebnisse beeinflusst, da in der Studie ausschließlich Studenten befragt wurden.

38 Cf. hierzu auch die Ausführungen bei Butterworth/Glawe (2011), in der die Salienzwerte der spirantisierten Stimuli in Westfalen bei 36,3 \% liegen, während sie in Brandenburg mit 92,5\% als hochsalient gelten. Die Stichprobe pro Region umfasste jeweils 16 Personen (cf. ibd.: 385 u. 382).

39 Ebenso Pabbe.
} 
bewerteten (cf. Elmentaler/Gessinger/Wirrer 2010: 117 u. 120). ${ }^{40}$ Gessinger merkt in einer früheren Studie an, dass die Lenisierungen von /p/ (Pabbe) und /t/ (bidde) für die SchleswigHolsteiner Gewährspersonen salient sind, Wegger jedoch nicht (cf. Gessinger 2008: 140141). ${ }^{41}$ Dies entspricht zwar nicht den hier ermittelten Salienzwerten für das lenisierte $/ \mathrm{k} /$, zu bemerken ist allerdings, dass diese Variante tendenziell von weniger Gewährspersonen als auffällig bewertet wurde als die anderen Lenisierungen.

Auffällig divergieren die Ergebnisse von Mihm und der vorliegenden Studie in Bezug auf das lenisierte /t/, das bei Mihm (Stimulus Mudder) mit $96 \%$ zu den besonders häufig erkannten Phänomenen zählt (im Vergleich zu $76 \%$ in dieser Untersuchung). Neben dem gewählten Stimulus spielt auch hier sicherlich die je nach Untersuchungsregion unterschiedliche Eigenund Fremdwahrnehmung der Varianten eine Rolle (cf. hierzu auch Elmentaler/Gessinger/Wirrer 2010: 119). Im Folgenden soll betrachtet werden, inwieweit sich Bremer und Hamburger Gewährspersonen in der Wahrnehmung regionaler Phänomene unterscheiden.

\subsection{Salienz und Region}

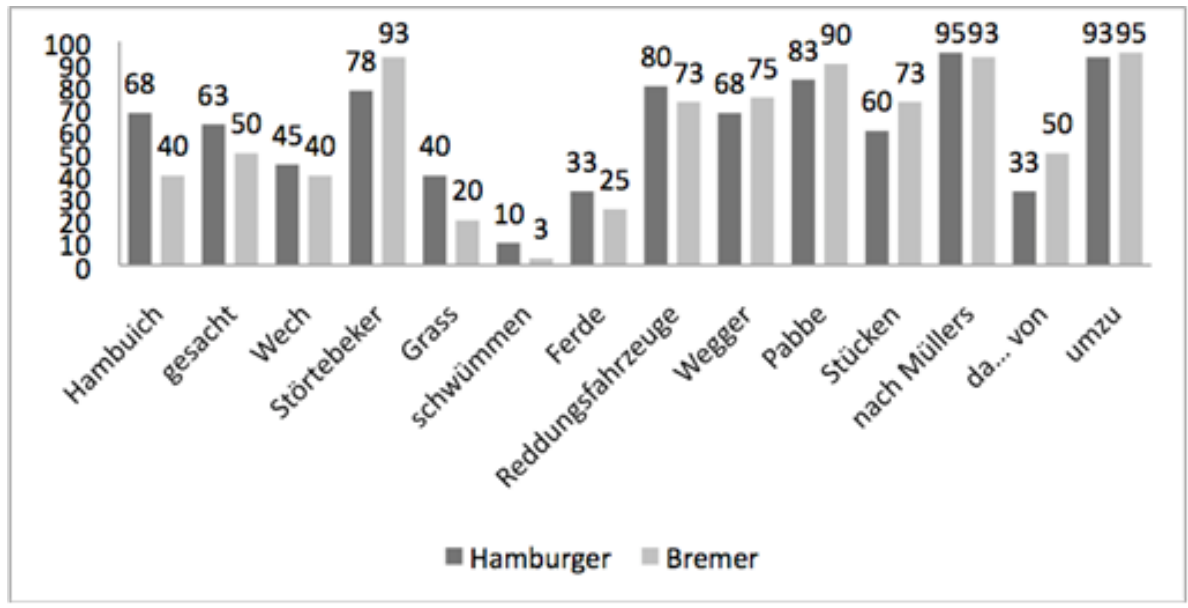

Abbildung 2: Wahrnehmung nach Region (Zahlen in Prozent).

Bei vielen Merkmalen bestehen, nach Herkunft der Gewährspersonen aufgefächert, nur geringe Unterschiede bzgl. ihrer Auffälligkeit, größere Differenzen gibt es jedoch bei der Wahrnehmung von Hambuich und Grass, die für die Hamburger salienter sind, während das alveolare/s/, die Pluralform Stücken sowie die Trennung des Pronominaladverbs mehr Bremern auffällt. Es stellt sich die Frage, ob einige dieser Merkmale je nach Stadt unterschiedlich in der Umgangssprache verbreitet sind, so dass es einerseits Varianten gibt, die aufgrund ihrer Fremdheit auffällig sind und andererseits Varianten, die Sprecher selbst gebrauchen, weniger salient sind, da sie nicht vom individuellen situativen Normhorizont abweichen (cf. Purschke 2011: 82). ${ }^{42}$ Erkenntnisse zu einer etwaigen regional unterschiedlichen Gebrauchsfrequenz bestimmter Varianten, die diese These stützen würden, können über die Auswertung der Objektdaten im Rahmen der qualitativen Studie gewonnen werden.

Im Folgenden sollen die Bewertung und die regionale Einordnung der Stimuli betrachtet werden. Diese divergieren allgemein stärker nach Region als die Salienz - auch ist zu

\footnotetext{
$40 \mathrm{Zu}$ diesen und auch den vorher dargestellten Werten ist $\mathrm{zu}$ bemerken, dass sie generell vorsichtig betrachtet werden müssen, da die Anzahl der Gewährspersonen aus den einzelnen Regionen zum Teil sehr gering ist (Schleswig Holstein: 18 GP, südlicher Niederrhein: 6 GP, Brandenburg-Berlin: 24 GP). Cf. zur Anzahl der Gewährspersonen Elmentaler/Gessinger/Wirrer 2010: 116-117.

4112 GP aus Schleswig-Holstein, 24 GP aus Berlin-Brandenburg.

42 Diese Annahme lässt sich jedoch schwer auf das Ergebnis des Stimulus S-törtebeker anwenden, da, wie angemerkt, dem Merkmal für Hamburg allgemein nur noch ein relikthafter Charakter zugesprochen wird.
} 
bemerken, dass Bremer und Hamburger (besonders morpho-syntaktische) Merkmale zum Teil unterschiedlich verorten.

\subsection{Bewertung und regionale Einordnung}

Bedingt durch die offene Fragestellung "Wie finden Sie es, wenn jemand so spricht"43 äußern die Befragten eine Vielzahl an Assoziationen, deren Einordnung in Kategorien sich nicht immer einfach gestaltet, da Gewährspersonen u. a. zwischen "beschreibenden und wertenden Kategorien" (Eichinger 2010: 437) wechseln. ${ }^{44}$ Für den vorliegenden Beitrag sollen zunächst schlaglichtartig explizit positive Bewertungen ("gut", "finde ich schön") und explizit negative Bewertungen ("furchtbar", "mag ich nicht") ausgewählter Stimuli betrachtet werden.

Tendenziell werden Phänomene der phonetisch/phonologischen und lexikalischen Ebene besser bewertet, als jene der morpho-syntaktischen, was sich mit der Annahme deckt, dass durch die schulische Erziehung bestimmte Phänomene zu Fehlerstereotypen werden und Elemente der Grammatik eher als korrekturbedürftig (und negativ) charakterisiert werden, als lautliche Merkmale. ${ }^{45}$ Allerdings sind nicht alle morpho-syntaktischen Phänomene stigmatisiert. Während der Stimulus nach Müllers gehen von $65 \%$ der Befragten negativ bewertet wird, werden die Formen Stücken und die Trennung von davon von nur ca. $24 \%$ der Befragten negativ konnotiert. 46

Am positivsten wird von den ausgewählten Merkmalen das alveolare /s/ bewertet. $47 \%$ der Befragten, die das Merkmal gehört haben, charakterisieren es u. a. als "schön", "süß" oder "symphatisch". Nur $9 \%$ äußern sich negativ, während die restlichen Befragten das Gehörte mit keiner besonderen Wertung belegen, sondern das Phänomen z. B. als "normal" oder "alltäglich" 47 bezeichnen. Auch Hambuich wird von über $40 \%$ der Gewährspersonen positiv konnotiert. ${ }^{48}$ Die Bewertung beider Merkmale stützt Auers Annahme, dass es sich bei beiden Phänomenen um nicht stigmatisierte Varianten handelt (cf. Auer 1998: 181), von denen eine, das alveolare /s/, sogar ein "hohes Prestige" (Auer 1998: 191) aufweist. Die hohe Salienz von S-törtebeker würde somit auch damit zusammenhängen, dass es sich um eine Variante handelt, die mit "einer hohen affektiv-evaluativen Ladung belegt" (Lenz 2010: 101) ist und von "vielen Sprechern metakommuniziert" (ibd.) wird. ${ }^{49}$

\footnotetext{
43 Es wurden somit keine Kontexte/Domänen vorgegeben. Vereinzelt differenzierten die Gewährspersonen jedoch eigenständig und kommentierten Stimuli mit Aussagen wie: "Bei Plattdeutschsprechern okay, bei anderen komisch."

44 Auch gestaltet es sich beispielsweise schwierig, Aussagen wie "Dieses Merkmal finde ich lustig" zu kategorisieren, da diese Ausdruck einer sowohl positiven als auch negativen Konnotation der Variante seitens der Sprecher/Hörer sein können.

45 Hierbei ist $\mathrm{zu}$ bemerken, dass nicht alle salienten morpho-syntakischen Merkmale grundsätzlich als korrekturbedürftig angesehen werden. (cf. Hettler 2013: 175).

46 Dieses Ergebnis deckt sich u. .a. mit der Annahme Blumes (2002: 19), dass die Verwendung der direktiven Präposition nach besonders vor Personennamen im Bewusstsein von Sprechern als schlimmer Regelverstoß gilt und gerne als Anlass für Sprachspott genommen wird.

47 Allerdings weisen auch fast $30 \%$ der Gewährspersonen in ihren Kommentaren darauf hin, dass diese Variante nur noch von älteren Personen gebraucht wird bzw. gar nicht mehr zu hören wäre.

48 Im Bereich der Spirantisierungen wird der Stimulus gesacht noch von $36 \%$ positiv bewertet, das spirantisierte $/ \mathrm{g} /$ in Wech dagegen nur von $11 \%$. Zur Erklärung dieser Differenzen erscheint es sinnvoll, in folgenden Auswertungen die metasprachlichen Kommentare der Gewährspersonen genauer zu analysieren sowie die Angaben zur Korrekturbedürftigkeit der Variante mit einzubeziehen. Auch Angaben zur situativen Angemessenheit von Varianten, wie sie im Rahmen des SiN-Projektes erhoben wurden, sind hier sicherlich von großer Relevanz.

${ }^{49}$ Hierfür spricht auch, dass das Auftreten und die Verbreitung der Variante von vielen Gewährspersonen während des Tests thematisiert werden.
} 
Interessant ist die divergierende Bewertung der unterschiedlichen Lenisierungen im Test. Diagramm 3 zeigt den Anteil explizit positiver sowie negativer Bewertungen an. Unter der Kategorie nicht wertend wurden Bewertungen vermerkt, die sich nicht ohne weiteres deuten ließen wie "weder sympathisch noch unsympathisch" und "normal" oder "Alltagssprache". 50 Gewertet wurden nur Aussagen von Personen, die die Variante kannten. ${ }^{51}$

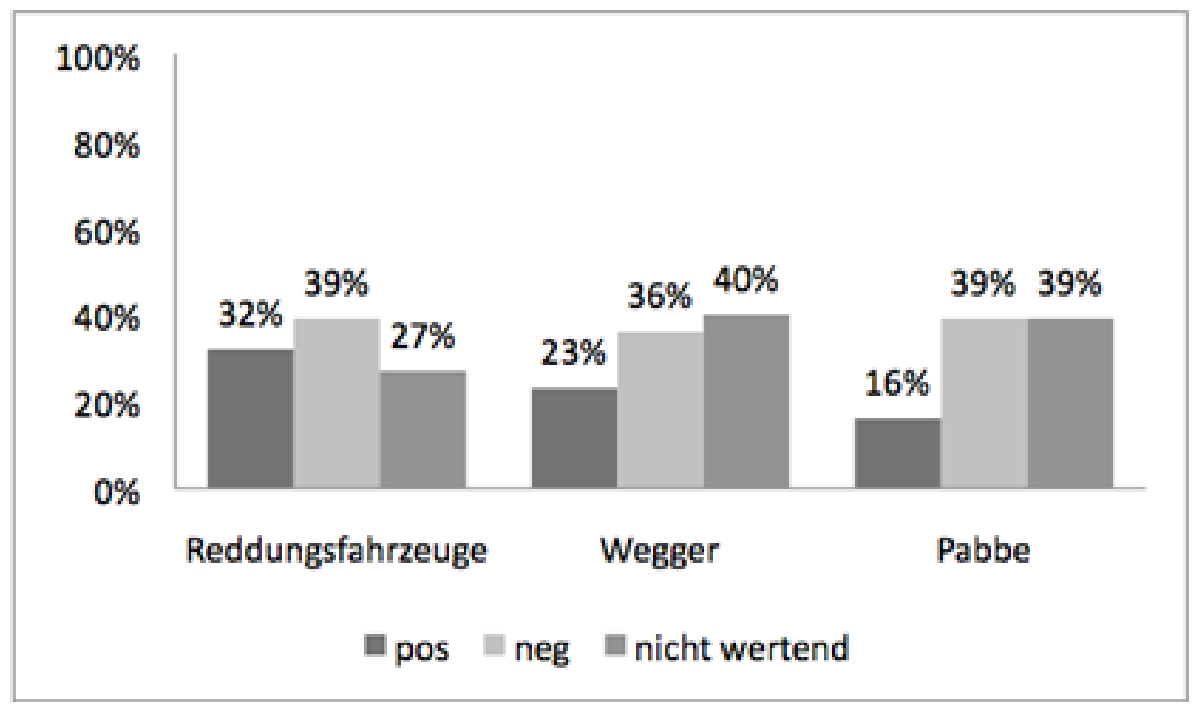

Abbildung 3: Bewertung der Lenisierungen gesamt. 52

Alle Lenisierungen werden von 36-39 \% der Befragten explizit negativ bewertet. So werden die Formen als "nachlässige Aussprache" oder "schlampige Jugendsprache" bezeichnet. Während die Stimuli Wegger und Pabbe zu einem fast ebenso großen Anteil auch als "normal" oder "in Ordnung" eingeordnet werden, wird der Stimulus Reddungsfahrzeuge von immerhin einem Drittel der Befragten positiv bewertet. So wird er des Häufigeren als "sympathisch" und "gut" charakterisiert, oft mit dem Hinweis, dass diese Aussprache "typisch für Hamburg" wäre. Diese Aussage spiegelt sich auch in der regionalen Verortung der Merkmale wieder, bei der eine Zuordnung zur eigenen Region häufig mit einer positiven Bewertung von Merkmalen korreliert.

In Diagramm 4 wird exemplarisch die Verortung der unterschiedlichen Stimuli mit lenisiertem Konsonanten nach "Hamburg", "Bremen", "Norddeutschland" und in "andere Regionen" betrachtet. ${ }^{53}$ Erneut wurden nur Angaben von Personen gewertet, die aussagten, dass Ihnen die Verwendung der Variante von anderen Sprechern (oder auch sich selbst) geläufig sei.

\footnotetext{
50 In weiterführenden Analysen müssen diese Bewertungen noch differenzierter kategorisiert und betrachtet werden.

51 Varianten, die den Gewährspersonen „unbekannt“" waren, wurden in der Regel als fremd und als negativ eingestuft. Angabe „Variante unbekannt“ bei den Stimuli: Reddungsfahrzeuge: 2, Wegger, 4, Pabbe: 8. Die Anzahl der GP, die den Prozentzahlen zugrunde liegt (cf. die Werte in Tabelle 1) ist: Reddungsfahrzeuge: 59 GP, Wegger: 63 GP, Pabbe: 61 GP.

52 Fehlende Werte zu 100 \%: Keine Angabe zur Bewertung bzw. Einordnung der Angabe nicht möglich.

53 Hierbei wurde die Angabe: "Merkmal ist in Hamburg und Bremen zu verorten" zu "Norddeutschland" gerechnet. Inwieweit das Konzept "Norddeutsch" auch die eigene Stadt miteinbezieht, wäre zu überlegen.
} 


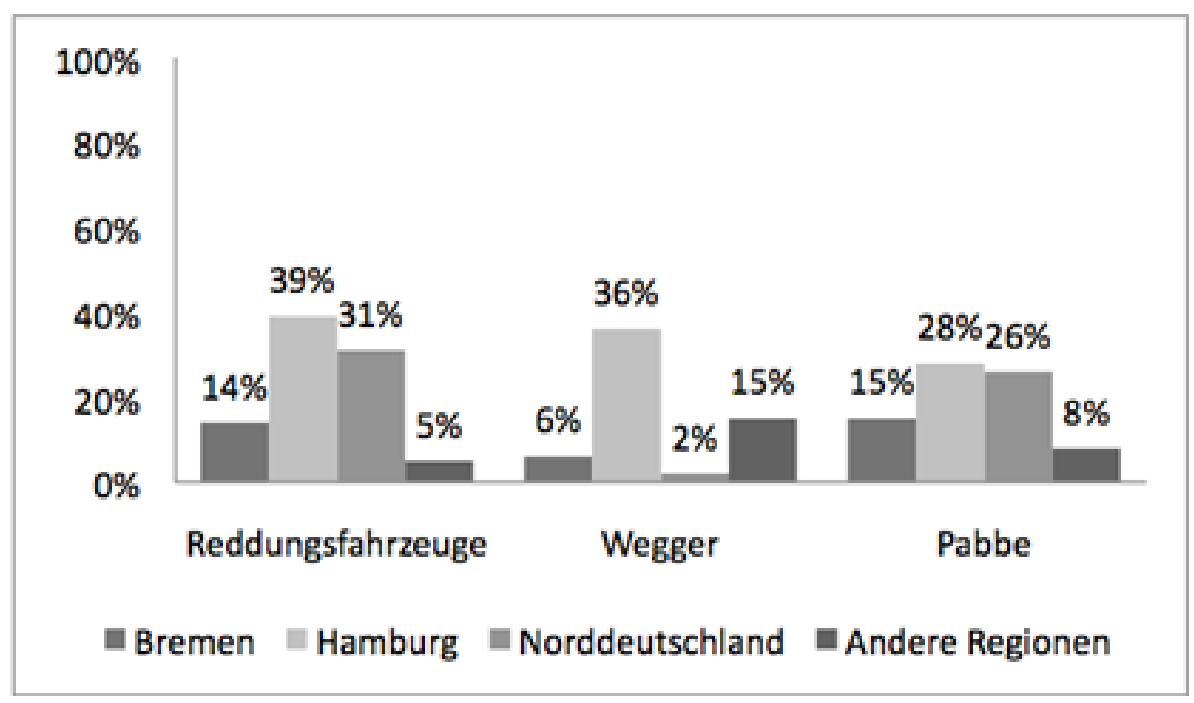

Abbildung 4: Regionale Einordnung der Lenisierungen. 54

Der Stimulus Reddungsfahrzeuge wird von insgesamt $84 \%$ der Befragten in den norddeutschen Raum eingeordnet, von etwa $40 \%$ der Befragten direkt nach Hamburg. Allgemein werden alle drei Stimuli mehrheitlich eher nach Hamburg als nach Bremen verortet, die Stimuli Wegger und Pabbe werden aber von einem gewissen Prozentsatz auch mit anderen Regionen wie beispielsweise "Hessen" oder dem "Osten", speziell "Sachsen" in Verbindung gebracht oder konnten (trotz Bekanntheit der Variante) von 10-13\% der Befragten gar nicht regional eingeordnet werden. Da die Verortung in andere Regionen auch häufig mit einer eher negativen Bewertung der Varianten verbunden ist, lässt sich annehmen, dass die im Vergleich positivere Bewertung des lenisierten /t/ auch mit dem Faktor Ortsloyalität verknüpft ist, zu dem u. a. Ziegler (1996: 154) ausführt: "Es ist davon auszugehen, dass die Anwesenheit bzw. das Fehlen von Ortsloyalität sowohl den Prozess der Wahrnehmung [...] als auch die Bewertung sprachlicher Erscheinungen sowie Verhaltensweisen entscheidend beeinflusst". 55

Anzumerken ist, dass die Hamburger Gewährspersonen die Lenisierungen häufiger der eigenen Stadt zuordnen als die Bremer, zum Teil selbst dann, wenn die Variante negativ bewertet wurde. Dies steht in Kontrast zur Bewertung und Einordnung der morpho-syntaktischen Merkmale, bei denen wiederum die Bremer bei allen Varianten eher angeben, dass diese Phänomene auch in ihrer Stadt zu hören wären, als die Hamburger, die lediglich die Form Stücken in stärkerem Maße für typisch Hamburgisch halten (cf. auch Hettler 2013: 177). ${ }^{56}$ Um festzustellen, ob die Hamburger allgemein phonologische Merkmale eher der eigenen Stadt zuordnen als die Bremer, ist es in weiteren Auswertungen erforderlich, auch die regional differenzierte Bewertung der anderen phonologischen Varianten zu betrachten. Aufschlussreich sind in diesem Zusammenhang sicherlich auch die Aussagen zum Korrekturverhalten und Normverständnis von Hamburgern und Bremern, wie sie im Rahmen der Interviews der qualitativen Untersuchung getätigt wurden.

\footnotetext{
${ }^{54}$ Fehlende Werte zu 100 \%: Trotz Bekanntheit der Einordnung keine regionale Verortung möglich, Verortung nicht nach Region, sondern sozial ("Die Unterschicht spricht so."), keine Angabe zur Verortung. Zu den möglichen Kategorien bei der Einordnung der Merkmale cf. auch Hettler (2013: 176).

55 Dem Einfluss von Ortsloyalität auf die Bewertung von Varianten kann in späteren Analysen im Rahmen des qualitativen Untersuchungsteils nachgegangen werden, da den 20 Gewährspersonen im Interview u. a. Fragen zur Einstellung gegenüber Bremen/Hamburg gestellt werden.

${ }^{56}$ Hier spielt sicher auch die eher positive Konnotation der Variante eine Rolle.
} 


\section{Qualitative Ergebnisse}

Neben der Korrelation von Salienz, Bewertung und regionaler Einordnung von Varianten ist zu untersuchen, inwieweit der individuelle Sprachgebrauch mit der Auffälligkeit von Merkmalen korreliert. ${ }^{57}$ Sind Varianten, die Sprecher im Interview, Freundesgespräch oder Vorlesetext selbst verwenden, für sie weniger salient als fremde Varianten? Im Folgenden sollen erste Ergebnisse von Type-Token-Analysen zweier Gewährspersonen vorgestellt werden, die zeigen, dass die Wahrnehmung von sprachlichen Phänomenen individuell abweichen kann. Betrachtet werden an dieser Stelle nur ausgewählte Merkmale der phonologischen Ebene.

\subsection{Gewährsperson HH-05}

Gewährsperson HH-05 ist ein gebürtiger Hamburger, Jahrgang 1940, besitzt Abitur und hat die längste Zeit seines Berufslebens im Bereich der Lehrerfortbildung gearbeitet. Im Salienztest nimmt er von den 33 Stimuli 18 wahr, also etwas mehr als die Hälfte. Um die gehörten Varianten mit deren Produktion in Verbindung zu setzen, wurde mittels einer Type-TokenAnalyse der prozentuale Anteil standarddivergenter Formen innerhalb der untersuchten Sprachproben der Gewährsperson ermittelt. In der folgenden Tabelle wird der (nicht nach den Settings aufgeschlüsselte) Gesamtwert gezeigt, hinter der Prozentzahl wird in absoluten Zahlen das Vorkommen der Standardvariante und der regionalen Variante aufgeführt. ${ }^{58}$

\begin{tabular}{|c|c|c|c|c|}
\hline Phänomen & gehört & $\begin{array}{l}\text { nicht } \\
\text { gehört }\end{array}$ & $\begin{array}{l}\text { Anteil } \\
\text { standarddivergenter } \\
\text { Realisierungen }\end{array}$ & $\begin{array}{l}\text { Beispiele } \\
\text { Realisierungen GP }\end{array}$ \\
\hline $\begin{array}{l}\text { Spirantisierung /g/ + } \\
\text { Vokalisierung /r/ bei - } \\
\text { burg }\end{array}$ & & $\mathrm{x}$ & $100 \%(0 / 18)$ & $\begin{array}{l}\text { Hambuich, } \\
\text { Mecklenbuich }\end{array}$ \\
\hline Spirantisierung /g/ & & $\begin{array}{l}\text { a) } x \\
\text { b) } x^{59}\end{array}$ & $70 \%(20 / 46)$ & $\begin{array}{l}\text { fraachten, } \\
\text { aufgesaacht, } \\
\text { Jungfernstiech }\end{array}$ \\
\hline Alveolares /s/ & $\mathrm{x}$ & & $7 \%(123 / 9)$ & $\begin{array}{l}\text { Hanses-tadt, S- } \\
\text { tichwort }\end{array}$ \\
\hline Vokalkürzung /a:/ & & $\mathrm{x}$ & $20 \%(4 / 1)$ & $\begin{array}{l}\text { Glass (Wein), } \\
\text { Fensterglass }\end{array}$ \\
\hline Rundung /i/ & & $\mathrm{x}$ & $4 \%(169 / 7)$ & $\begin{array}{l}\text { Müschung, } \\
\text { inzwüschen }\end{array}$ \\
\hline $\begin{array}{l}\text { Ausfall } \\
\text { Affrikatenkomponente }\end{array}$ & & $\mathrm{x}$ & $88 \%(1 / 7)$ & Fund (vs. Pfund) \\
\hline Lenisierung $/ \mathrm{t} /$ & $\mathrm{x}$ & & $55 \%(65 / 79)$ & $\begin{array}{l}\text { Vadder, } \\
\text { Ridderstraße, } \\
\text { Mudder }\end{array}$ \\
\hline Lenisierung /k/ & $\mathrm{X}$ & & $18 \%(14 / 3)$ & Akademigger \\
\hline Lenisierung /p/ & $\mathrm{x}$ & & $5 \%(18 / 1)$ & rebbarieren \\
\hline
\end{tabular}

Tabelle 2: GP HH-05.

57 Grundsätzlich wäre in weiteren Analysen zu diskutieren, in welche Richtung sich Perzeption und Sprachgebrauch aufeinander auswirken. Sind Varianten auffällig bzw. unauffällig, weil sie eine bestimmte Frequenz in der regionalen Umgangssprache aufweisen oder hat, wie $u$. a. Purschke annimmt, die (positive oder negative) Wahrnehmung von regionalen Merkmalen "kommunikative Konsequenzen" (Purschke 2011: 82) und wirkt sich auf den Sprachgebrauch (z. B. im Sinne der Vermeidung von Varianten) aus?

$58 \mathrm{Zu}$ bemerken ist, dass es sich um vorläufige Ergebnisse handelt. In späteren Analysen sollen u. a. einige Varianten je nach Lautkontext aufgefächert dargestellt und behandelt werden.

59 Die Angaben gelten für die Stimuli a) gesacht und b) Wech. 
Auffällig ist der Befund bei den Spirantisierungen - so realisiert der Befragte eine Spirantisierung von /g/ + Vokalisierung von /r/ durchgehend bei allen Namen, die auf -burg enden, wie auch in Harbuich oder Mecklenbuich. Auch der Ausfall der Affrikatenkomponente ist relativ durchgängig. Vereinzelt wir das /s/ alveolar realisiert, es ist jedoch kein (z. B. lexemgebundenes) Muster zu erkennen. ${ }^{60} \mathrm{Bzgl}$. der Lenisierungen erweicht die Gewährsperson am häufigsten $/ \mathrm{t} / \mathrm{zu} / \mathrm{d} / \mathrm{-}$ Lenisierungen von $/ \mathrm{k} /$ oder $/ \mathrm{p} /$ treten weniger häufig auf. Abgesehen von den Lenisierungen und dem alveolaren /s/ wird ein Großteil der Merkmale, die von der Gewährsperson in den Sprachproben realisiert werden, nicht gehört. Dieses erste Ergebnis, das einen Zusammenhang zwischen der Perzeption und der Produktion von regionalen Merkmalen annehmen lässt, muss jedoch mit Vorsicht betrachtet werden, wie die Ergebnisse einer zweiten Hamburger Gewährsperson zeigen.

\subsection{Gewährsperson HH-01}

\begin{tabular}{|l|l|l|l|l|}
\hline Phänomen & gehört & $\begin{array}{l}\text { nicht } \\
\text { gehört }\end{array}$ & $\begin{array}{l}\text { Anteil } \\
\text { standarddivergenter } \\
\text { Realisierungen }\end{array}$ & $\begin{array}{l}\text { Beispiele } \\
\text { Realisierungen GP }\end{array}$ \\
\hline $\begin{array}{l}\text { Spirantisierung/g/ + } \\
\text { Vokalisierung/r/ bei - } \\
\text { burg }\end{array}$ & $\mathrm{x}$ & & $89 \%(1 / 8)$ & Hambuich \\
\hline Spirantisierung/g/ & & $\begin{array}{l}\text { a) } \mathrm{x} \\
\mathrm{b}) \mathrm{x}\end{array}$ & $85 \%(10 / 56)$ & gesacht, Fußwech \\
\hline Alveolares /s/ & & $\mathrm{x}$ & $1 \%(97 / 1)$ & s-tufen (Verb) \\
\hline Vokalkürzung /a:/ & & $\mathrm{x}$ & $50 \%(2 / 2)$ & $\begin{array}{l}\text { Glass (Wein) } \\
\text { Fensterglass }\end{array}$ \\
\hline Rundung /i/ & $\mathrm{x}$ & & $8 \%(132 / 12)$ & ürgendwie \\
\hline $\begin{array}{l}\text { Ausfall } \\
\text { Affrikatenkomponente }\end{array}$ & $\mathrm{x}$ & $\mathrm{x}$ & $90 \%(1 / 9)$ & Ferd \\
\hline Lenisierung /t/ & $\mathrm{x}$ & & $42 \%(7 / 5)$ & $\begin{array}{l}\text { Hängemadde, } \\
\text { Vadder }\end{array}$ \\
\hline Lenisierung /k/ & $\mathrm{x}$ & & $0 \%(14 / 0)$ & Landungsbrüggen \\
\hline Lenisierung /p/ & & &
\end{tabular}

Tabelle 3: GP HH-01.

Auch Gewährsperson HH-01 ist männlich, Jahrgang 1940 und hat Abitur. Der Befragte hat zuletzt als Musiker gearbeitet. Wie Gewährsperson HH-05 hat er 18 der 33 Merkmale erkannt - allerdings zum Teil andere Phänomene.

Bzgl. der Produktion standarddivergenter Varianten ähnelt HH-01 der anderen Gewährsperson, Abweichungen bestehen in der vereinzelten Verwendung der standardsprachlichen Variante "Hamburg", sowie in einem höheren Auftreten von lenisiertem /k/. Für standarddivergente Realisierungen im Sinne von Pabbe gibt es keine Belege. Bzgl. der Perzeption werden, ähnlich wie bei Gewährsperson HH-05, die Lenisierungen gehört, die Spirantisierung in gesacht sowie die Vokalkürzung in Grass jedoch nicht. Abweichend von Gewährsperson HH-05 werden die Formen Hambuich und schwümmen jedoch als standarddivergent eingeordnet, obwohl die Gewährsperson selbst auch die Variante Hambuich verwendet. Bzgl. des Vorkommens von gerundetem /i/ ist zu bemerken, dass dieses, anders als bei HH-05, nur lexemgebunden (ürgendwie) auftritt. Auffällig bei der Gewährsperson HH-01 ist, dass sie (ausgehend von den

${ }^{60} \mathrm{Zu}$ bemerken ist, dass die Gewährsperson das Phänomen als hamburgisches Merkmal nennt, jedoch angibt, es selbst nicht zu verwenden. 
Ergebnissen der quantitativen Untersuchung) ein wenig salientes Phänomen wie die Rundung von /i/ bemerkt, das hochsaliente alveolare /s/ jedoch nicht. ${ }^{61}$

Erste Analysen weiterer Gewährspersonen zeigen, dass Parameter wie das Alter, aber auch Faktoren wie das Sprachwissen, die Ortsfestigkeit oder die Einstellung zum Hamburgischen bzw. Bremischen, eine größere Rolle als der eigene Sprachgebrauch in Bezug auf die Perzeption von Merkmalen spielen können. ${ }^{62}$ Im Fall von Gewährsperson HH-01 kann z. B. der Umstand, dass der Befragte seit drei Jahren zeitweise in Lübeck lebt, häufiger in Berlin ist und während des Interviews Vergleiche zwischen der Sprache in den Orten zieht, dafür sorgen, dass er im Gegensatz zu HH-05 gewisse Formen eher wahrnimmt. Weiterführende inhaltsbezogene Analysen der Interviews und der metasprachlichen Kommentare der Gewährspersonen während des Salienztests sind nötig, um weitere Aufschlüsse über individuelle Gründe für die unterschiedliche Wahrnehmung von Phänomenen zu finden und, soweit möglich, etwaige Perzeptionsmuster zu finden. Durch die qualitative Untersuchung soll zudem auch ein Beitrag zur Bremer und Hamburger Stadtsprachenforschung in dem Sinne geleistet werden, dass durch die Analyse der objektiven Daten, also der Sprachaufnahmen, zumindest exemplarisch überprüft werden kann, inwieweit bestimmte Varianten auftreten.

\section{$6 \quad$ Fazit und Ausblick}

Bezüglich der Frage, in welchem Maße Faktoren wie die Gebrauchsfrequenz und die Bewertung von Varianten die Salienz von Merkmalen bedingen, weisen die hier vorgestellten ersten Ergebnisse darauf hin, dass je nach Merkmal eine unterschiedliche Korrelation innerund außersprachlicher Faktoren für eine höhere oder niedrigere Auffälligkeit verantwortlich ist und bestätigen somit die bestehende Annahme, dass die Salienz von Merkmalen keinesfalls ein statisches Konzept ist. So können saliente Phänomene sowohl stigmatisiert (nach Müllers) als auch besonders positiv besetzt sein (Hambuich) und in Bezug auf ihre Gebrauchsfrequenz in der Alltagssprache mehr (Lenisierung /t/) oder weniger stark (alveolares /s/) ausgeprägt sein. Im Vergleich mit den Ergebnissen anderer Studien scheinen zudem die lexikalische Einbettung von Stimuli sowie die regionale Herkunft von Sprechern bzw. Hörern die Auffälligkeit von Merkmalen zu beeinflussen. Die "dynamische Größe" (Lenz 2010: 101) von Salienz zeigt sich auch im individuellen Vergleich von Sprechern: Die Perzeption regionaler Merkmale korreliert nicht zwingend mit der Produktion bzw. Vermeidung derselben, sondern kann auch sprachbiografische Gründe haben. Extralinguistische Faktoren, wie z. B. der Einfluss des Berufs und des Alters auf die Wahrnehmung von Merkmalen, müssen noch untersucht werden. Davon ausgehend könnten Gewährspersonen mit ähnlichen Perzeptionsmustern sowie sprachlichen und (sprach)biografischen Gemeinsamkeiten zu bestimmten Hörer- bzw. Sprechertypen zusammengefasst werden.

Grundsätzlich stellt sich die Frage, ob sich Salienz als einheitliches Phänomen darstellt oder ob vielmehr unterschiedliche Faktorenbündel die Auffälligkeit von Merkmalen bedingen. Im Rahmen einer Erweiterung des Salienzbegriffs wäre es in folgenden Auswertungen deswegen sinnvoll, Varianten mit einer ähnlichen Kombination von Parametern, die ihre Salienz stützen (oder hemmen), zu gruppieren und zu kategorisieren. Denkbar wären Merkmalsgruppen, die

\footnotetext{
61 Eine Erklärung hierfür könnte sein, dass der Fokus der Gewährsperson auf einem anderen Satzteil lag - so wurde von HH-01 die Realisierung von Ort in dem Salienztestsatz als standarddivergent eingeordnet. Cf. zur Perzeption nicht-intendierter Merkmale u. a. Elmentaler/Gessinger/Wirrer 2010: 134).

62 Cf. hierzu auch Lenz: "Die Fähigkeit, eigene wie fremde sprachliche Charakteristika zu beurteilen, ist bspw. abhängig von der individuellen Fähigkeit zur Reflexions- und Selbstwahrnehmung [...] oder von der Häufigkeit und Art sprachlicher Kontakte" (Lenz 2003: 387). Denkbar wäre beispielsweise, dass Sprecher um die Auffälligkeit selbst verwendeter regionaler Merkmale wissen und auf diese auch besonders achten. In weiteren Analysen erscheint es deswegen sinnvoll, besonders die Angaben zu Charakteristika der eigenen Sprache, wie sie im Rahmen der Interviews getätigt wurden, in Bezug zu den Salienztestergebnissen zu setzen.
} 
aufgrund ihrer hohen Frequenz nicht auffällig sind, und solche, deren Salienz - unabhängig von ihrer Frequenz - hauptsächlich von der Bewertung und dem Ausmaß, in dem sie z. B. von Sprechern metakommunikativ thematisiert werden, beeinflusst wird. Auch wäre im Vergleich mit anderen Studien zu untersuchen, ob die Herkunft des Hörers auf die Salienz aller Varianten Einfluss hat oder ob Merkmale existieren, die unabhängig von der Region (z. B. aufgrund merkmalsintrinsischer Faktoren) als salient empfunden werden.

\section{Literatur}

Auer, Peter (1998): "Hamburger Phonologie. Eine variationslinguistische Skizze zur Sprache der Hansestadt heute". Zeitschrift für Dialektologie und Linguistik 65: 179-197.

Blume, Herbert (2002): "'Ich gehe nach Kröpcke." - Der Funktionswandel der lokalen Präposition "nach" im norddeutschen Hochdeutsch vom 18. bis ins 21. Jahrhundert, vor seinem niederdeutschen Hintergrund betrachtet". In: Hübener, Andrea/Unglaub, Erich (eds.): Kuriosum als Erkenntnis. Festschrift für Leif Ludwig Albertsen. Flensburg, Futura Edition: 13-21.

Butterworth, Judith/Glawe, Meike (2011): "'Wir sprechen hier an und für sich reinrassiges Hochdeutsch". Zur Erforschung der subjektiven Seite von Sprachverwendung". In: Ganswindt, Brigitte/Purschke, Christoph (eds.): Perspektiven der Variationslinguistik. Beiträge aus dem Forum Sprachvariation. Hildesheim/Zürich/New York, Olms: 371-393. (= Germanistische Linguistik 216/217).

Dingeldein, Heinrich J. (1994): "Befragungen zum Sprachgebrauch als Problem der Dialektologie". In: Mattheier Klaus J./Wiesinger, Peter (eds.): Dialektologie des Deutschen. Forschungsstand und Entwicklungstendenzen. Tübingen, Niemeyer: 393-411.

Dudenredaktion (ed.) (2005): Das Aussprachewörterbuch. Band 6. 6., überarbeitete und aktualisierte Auflage. Mannheim etc.: Dudenverlag.

Dudenredaktion (ed.) (2009): Die Grammatik. Band 4. 8., überarbeitete Auflage. Mannheim etc.: Dudenverlag.

Eichinger, Ludwig (2010): "Kann man der Selbsteinschätzung von Sprechern trauen?" In: Anders, Christina A./Hundt, Markus/Lasch, Alexander (eds.): Perceptual Dialectology. Neue Wege der Dialektologie. Berlin/New York, de Gruyter: 433-449. (= Linguistik Impulse und Tendenzen 38).

Elmentaler, Michael et al. (2006): "Sprachvariation in Norddeutschland. Ein Projekt zur Analyse des sprachlichen Wandels in Norddeutschland". Osnabrücker Beiträge zur Sprachtheorie 71: 159-178.

Elmentaler, Michael/Gessinger, Joachim/Wirrer, Jan (2010): "Qualitative und quantitative Verfahren in der Ethnodialektologie von Salienz". In: Anders, Christina A./Hundt, Markus/Lasch, Alexander (eds.): Perceptual Dialectology. Neue Wege der Dialektologie. Berlin/New York, de Gruyter: 111-149. (= Linguistik - Impulse und Tendenzen 38).

Gessinger, Joachim (2008): "Perzeptive Dialektologie und Spracheinstellungsforschung". Niederdeutsches Jahrbuch 131: 133-144.

Herrgen, Joachim/Schmidt, Jürgen Erich (1985): "Systemkontrast und Hörerurteil. Zwei Dialektalitätsbegriffe und ihre entsprechenden Meßverfahren". Zeitschrift für Dialektologie und Linguistik 52/1: 20-42.

Herrmann-Winter, Renate (1977): "Soziolinguistische Aspekte empirischer Erhebungen zur sprachlichen Varianz". In: Hartung, Wolfdietrich (ed.): Normen in der sprachlichen Kommunikation. Berlin, Akademie-Verlag: 209-247. (= Sprache und Gesellschaft 11).

Hettler, Yvonne (2008): Hamburgs Stadtsprache und ihre salienten Merkmale in der Wahrnehmung der Sprecher. Hamburg. Magisterarbeit.

Hettler, Yvonne (2013): "'Nach Müllers geht man nicht!" Zur Salienz und Bewertung morphosyntaktischer Phänomene in Bremen und Hamburg". In: Hettler, Yvonne/Jürgens, Carolin/Langhanke, Robert/Purschke, Christoph (eds.): Variation, Wandel, Wissen. Studien 
zum Hochdeutschen und Niederdeutschen. Frankfurt a. M. etc., Peter Lang: 161-183. (= Sprache in der Gesellschaft 32).

Hundt, Markus (1992): Einstellungen gegenüber dialektal gefärbter Standardsprache. Eine empirische Untersuchung zum Bairischen, Hamburgischen, Pfälzischen und Schwäbischen. Stuttgart: Franz Steiner. (= Zeitschrift für Dialektologie und Linguistik Beihefte 78).

Kellner, Klaus (2011): Bremisches Wörterbuch. Was man in Bremen so sacht. Vergnügliche Erläuterungen. 2. Auflage. Bremen/Boston: Kellner.

Kiesewalter, Carolin (2011): "Zur Salienz remanenter Merkmale des Neuhessischen". In: Ganswindt, Brigitte/Purschke, Christoph (eds.): Perspektiven der Variationslinguistik. Beiträge aus dem Forum Sprachvariation. Hildesheim/Zürich/New York, Olms: 335-369. (= Germanistische Linguistik 216/217).

Lasch, Miguel (1989): "Aussprache von /\# sp/ und /\# st/ in der Hamburger Umgangssprache". Korrespondenzblatt des Vereins für niederdeutsche Sprachforschung 96: 11-14.

Lenz, Alexandra (2003): Struktur und Dynamik des Substandards. Eine Studie zum Westmitteldeutschen (Wittlich/Eifel). Stuttgart: Franz Steiner. (= Zeitschrift für Dialektologie und Linguistik Beihefte 125).

Lenz, Alexandra (2010): "Zum Salienzbegriff und zum Nachweis salienter Merkmale". In: Anders, Christina A./Hundt, Markus/Lasch, Alexander (eds.): Perceptual dialectology. Neue Wege der Dialektologie. Berlin/New York, de Gruyter: 89-111. (= Linguistik Impulse und Tendenzen 38).

Lorenz, Cornelia (2014): "Salienz unter Einheimischen und Zugezogenen - ein Vergleich". Linguistik online 66: 137-146.

Martens, Peter (1981): "Hamburgisch. Geest-Mundart, Marsch-Mundart, Missingsch". In: Inter Nationes (ed.): Deutsche Dialekte. 9 Dialekte der Bundesrepublik Deutschland. Bonn, Inter Nationes: $257-388$.

Mihm, Arend (1985): "Prestige und Stigma des Substandards. Zur Bewertung des Ruhrdeutschen im Ruhrgebiet". In: Mihm, Arend (ed.): Sprache an Rhein und Ruhr. Dialektologische und soziolinguistische Studien zur sprachlichen Situation im Rhein-RuhrGebiet und ihrer Geschichte. Stuttgart, Steiner Verlag Wiesbaden: 163-194. (=Zeitschrift für Dialektologie und Linguistik Beihefte 50).

Mihm, Arend (2000): "Die Rolle der Umgangssprachen seit der Mitte des 20. Jahrhunderts". In: Besch, Werner et al. (eds.): Sprachgeschichte. Ein Handbuch zur Geschichte der deutschen Sprache und ihrer Erforschung. 2. Teilband. 2., vollständig neu bearbeitete und erweiterte Auflage. Berlin/New York, de Gruyter: 2107-2137.

Purschke, Christoph (2011): Regionalsprachlichkeit und Hörerurteil. Grundzüge einer perzeptiven Variationslinguistik. Stuttgart: Franz Steiner. (= Zeitschrift für Dialektologie und Linguistik Beihefte 149).

Scheel, Käthe (1963): "Hamburger Missingsch". In: Simon, Werner/Bachofer, Wolfgang/Dittmann, Wolfgang (eds.): Festgabe für Ulrich Pretzel zum 65. Geburtstag dargebracht von Freunden und Schülern. Berlin, E. Schmidt: 381-390.

Schmidt, Jürgen Erich (2005): "Die deutsche Standardsprache: eine Varietät - drei Oralisierungsnormen". In: Eichinger, Ludwig M./Kallmeyer, Werner (eds.): Standardvariation - Wie viel Variation verträgt die deutsche Sprache? Berlin/New York, de Gruyter: 278-305.

Schirmunski, Viktor M. (1928/1929): "Die schwäbischen Mundarten in Transkaukasien und Südukraine". Teuthonista 5: 38-60, 157-171.

Schirmunski, Viktor M. (1930): "Sprachgeschichte und Siedlungsmundarten". Germanischromanische Monatsschrift XVIII: 113-122, 171-188.

SiN: DFG-Projekt "Sprachvariation in Deutschland". http://www.corpora.uni-hamburg.de /sin/index.html, letzter Zugriff am 23.04.2014. 
Spiekermann, Helmut (2008): Sprache in Baden-Württemberg. Merkmale des regionalen Standards. Tübingen: Niemeyer. (=Linguistische Arbeiten 526).

Stellmacher, Dieter (1977): Studien zur gesprochenen Sprache in Niedersachsen. Eine soziolinguistische Untersuchung. Marburg: Elwert. (= Deutsche Dialektgeographie 82).

Stellmacher, Dieter (1981): Niedersächsisch. Düsseldorf: Schwann. (= Dialekt/Hochsprache kontrastiv Heft 8).

Trost, Pavel (1968): "Primäre und sekundäre Dialektmerkmale". In: Schmitt, Ludwig E. (ed.): Verhandlungen des 2. internationalen Dialektologenkongresses. Band 2. Wiesbaden, Franz Steiner: 823-826.

Trudgill, Peter (1986): Dialects in Contact. Oxford: Blackwell. (=Language in Society 10).

Ziegler, Evelyn (1996): Sprachgebrauch, Sprachwissen, Sprachvariation. Eine Familienfallstudie. Frankfurt a. M. etc.: Lang. (=Variolingua 2). 


\section{Anhang}

Vorlesetext

Besuchen Sie Husum

Seit vielen Jahren ist Husum ein Geheimtipp unter Ausflüglern. Von Hamburg aus gelangt man mit der Bahn in ca. zwei Stunden in dieses Städtchen, in dem es viel zu sehen gibt. So hat Theodor Storm in Husum den Schimmelreiter vollendet - eine steinerne Statue mit Pferd und Reiter gibt es zwar nicht, dafür lohnt sich aber ein Besuch des Theodor-Storm-Zentrums. Bei einer Stadtführung können Sie viel über die Geschichte Husums erfahren - so z. B. über den Butterkrieg, der im Jahr 1848 ausbrach, als ein Händler die gesamte Butter in Husum aufkaufte, um sie nach Helgoland zu schicken. Lustig geht es im "Puppenspieler Museum" zu, in dem Figuren aus Pappe und Holz von Künstlern aus aller Welt bestaunt werden können. An Markttagen herrscht auf dem Husumer Wochenmarkt ein geschäftiges Treiben und viele Ausflügler nutzen an warmen Tagen die Chance, günstig ein paar Kirschen zu kaufen, um sie im Gras liegend im Schlosspark zu essen. Im März können Sie dort auch das Krokusblütenfest erleben, von dem gesagt wird, dass es das "Wunder des Nordens" wäre. In der Nähe des Marktplatzes befindet sich die Kirche St. Marien. Berühmt ist sie für ein Halbrund über dem Altar, das durch das Licht eines dahinter liegenden Glasfensters wie ein Regenbogen erstrahlt. Das Kirchlein am Meer im Stadtteil Schobüll soll um 1240 von drei frommen Mädchen gestiftet worden sein. Das Gebäude gilt als eine der schönsten Kirchen in Schleswig-Holstein und wird gerne genutzt, um Hochzeiten zu feiern. Auch das Umland Husums ist sehenswert und ein Paradies für Pferdeliebhaber und Wanderer. Wer schon immer einen Hahn als Wecker haben wollte, kann auf ausgewählten Höfen übernachten und sogar einen Treckerführerschein machen! Und wenn Sie aufgrund der guten Nordseeluft Appetit bekommen: In den Meiereien und Käsereien in Nordfriesland finden sie herzhafte Milchprodukte - drei Stationen der Käsestraße Schleswig-Holstein liegen ganz in der Nähe. Bevor man sich wieder auf den Rückweg macht, sollte man sich unbedingt ein Fischbrötchen am Hafen kaufen oder dort in einem der vielen Restaurants den Tag bei einem guten Glas Wein ausklingen lassen. Husum ist immer eine Reise wert! 